\title{
Cytomegalievirus bei Nierentransplantation - Diagnose, Prävention und Management
}

\author{
Das Cytomegalievirus (CMV) ist die häufigste virale Infektion, die nach der Transplantation \\ solider Organe zu schwerwiegenden Komplikationen führen kann. Eine deutsche Expertengruppe \\ hat nun die im Jahr 2013 veröffentlichten internationalen Konsensus-Leitlinien zu CMV bei \\ Transplantation solider Organe zusammengefasst und bewertet.
}

Autoren

Volker Kliem

Martina Sester

Martin Nitschke

Burkhard Tönshoff

Klemens Budde

Ingeborg A. Hauser

Michael Schmitt

Britta Höcker

Oliver Witzke
Abb. 1 Der CMV-Serostatus von Spender (D) und Empfänger ( $\mathrm{R}$ ) dient der Risikoabschätzung. Die Abbildung gibt die Risiken ohne prophylaktische oder präemptive Maßnahmen wieder [28].

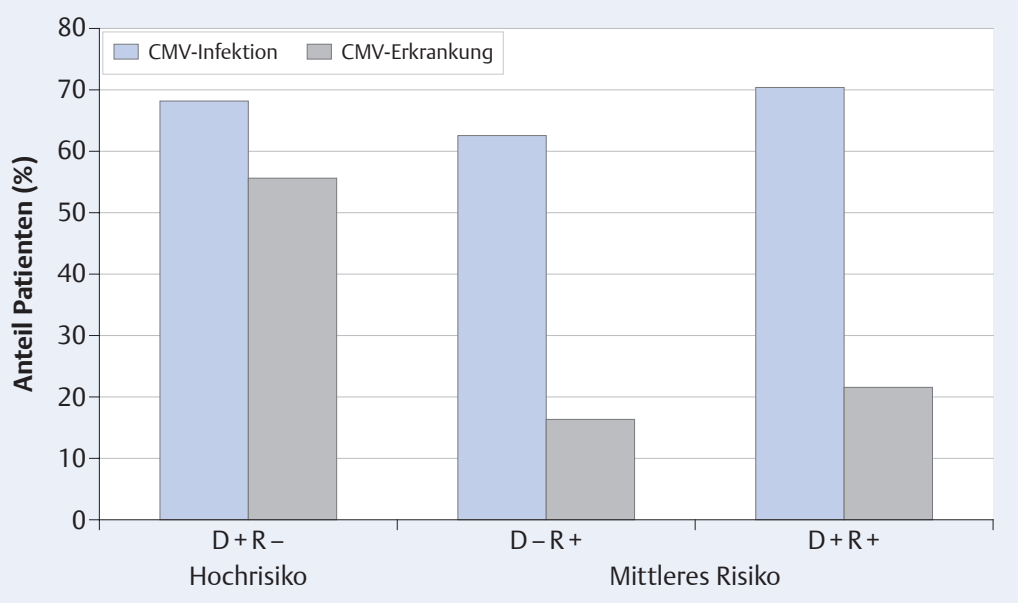
gruppe hat das Update zum Anlass genommen, um spezifische Aspekte und Fragestellungen für die praktische Anwendung bei Nierentransplantationen in Deutschland zu adressieren. Die
Empfehlungen der deutschen Expertengruppe sind wie folgt gekennzeichnet:

- hoher Übereinstimmungsgrad: mehrheitlicher Konsens zu den diskutierten Themen

- geringer Übereinstimmungsgrad: differierende Meinungen zu der jeweiligen Thematik. Die Aussagen wurden jedoch von der Mehrheit der Autoren getragen.

nach Transplantation reaktiviert werden. Eine CMV-Erkrankung kann

- unspezifisch sein, d.h. mit Fieber, Leuko- und Thrombozytopenie einhergehen, aber auch

- zu diversen Organmanifestationen führen [5, 20, 27, 32-34].

Durch eine zuverlässige Diagnostik und eine konsequente Behandlung kann die CMV-assoziierte erhöhte Morbidität und Mortalität nach Transplantation signifikant gesenkt werden.

Internationaler Konsensus | Im August 2013 wurde ein Update der Internationalen Konsensus -Leitlinien zum Management des CMV bei Transplantation solider Organe publiziert. Es widmet sich sich der Diagnostik, Immunologie, Prävention, Behandlung, Arzneimittelresistenz und pädiatrischen Aspekten [15]. Eine deutsche Experten-

\section{Diagnostik}

Vor der Transplantation I Vor einer Transplantation sollte die CMV-Serologie von Spender und Empfänger bestimmt werden. Bei Transplantatempfängern (R), die bislang noch keinen Kontakt mit dem Cytomegalievirus hatten (seronegativ für CMV-spezifische Antikörper) und das Organ eines CMV-seropositiven Spenders (D) übertragen bekommen, ist das Risiko für infektionsbedingte Komplikationen besonders hoch. Es liegt eine sogenannte D+/R--Serokonstellation vor ( Abb. 1). Der passive Transfer von Antikörpern durch die Gabe von Blutprodukten oder die Präsenz maternaler Antikörper bei Neugeborenen kann vorübergehend $\mathrm{zu}$ falsch-positiven serologischen Testergebnissen führen; dies muss beachtet und der Patient im Zweifelsfall als CMV-seronegativ eingestuft werden. Neben dem Serostatus (humorale Immunität) kann die Bestimmung der zellulären Immunität, z.B. die Messung CMV-spezifischer T-Zellen, das Immunmonitoring erweitern [25].

Nach der Transplantation | Nach einer Transplantation sollten ausschließlich quantitative Verfahren, bevorzugt über PCR, angewendet werden (aus Vollblut und aus Plasma). Die Bestimmung CMVspezifischer T-Zellen ist ein neuer interessanter experimenteller Forschungsansatz, um die individuelle spezifische Immunantwort auf das CMV besser abschätzen zu können [u.a. 30].

Grenzwerte I Bisher sind keine standardisierten Grenzwerte verfügbar, sodass die Assays nicht vergleichbar sind. Daher sollten bei einem Patienten - weder das Probenmaterial (Plasma/Vollblut) - noch das Labor 
gewechselt werden und Grenzwerte sollten in Kollaboration mit dem Labor ermittelt werden (hohe Übereinstimmung). Zukünftig werden zumindest die quantitativen PCR-Verfahren durch einen neuen internationalen WHO-Referenzstandard besser standardisiert werden können.

Gewebs-invasive CMV-Erkrankung I Die Diagnose einer Gewebs-invasiven CMV-Erkrankung basiert auf dem CMV-Nachweis im Biopsat. Die bevorzugte Nachweismethode ist die Histologie oder die DNA-Hybridisierung in situ $[11,17,19]$. Bei gastrointestinalem CMV-Befall kann die Testung auf CMV mittels quantitativer PCR im peripheren Blut negativ ausfallen, selten auch bei einer CMVPneumonitis $[6,35]$.

Die Experten empfehlen dringend, bei Patienten nach Nierentransplantation mit klinischen Symptomen oder dem Verdacht auf eine gastrointestinale CMV-Erkrankung (z. B. persistierende Durchfälle, Magenbeschwerden) eine endoskopische Diagnostik inklusive einer Biopsie durchzuführen. Das Biopsat sollte von einen in der CMV-Diagnostik erfahrenen Pathologen beurteilt werden ( $\triangleright$ Abb. 2).

\section{Prophylaxe und präemptive Therapie}

Zwei Strategien | Zur Prävention der CMV-Infektion sind prinzipiell zwei Strategien möglich:

- Prophylaxe: Verabreichen einer antiviralen Medikation über einen definierten Zeitraum, um der Virusvermehrung entgegenzuwirken

- Präemptive Therapie: Engmaschiges diagnostisches Monitoring der Viruslast, um eine CMVReplikation frühzeitig und möglichst noch vor Auftreten von Symptomen zu detektieren und zeitnah eine antivirale Therapie einzuleiten

Beide Strategien werden als praktikable Ansätze zur Prävention einer CMV-Erkrankung empfohlen (hohe Übereinstimmung). Die Wahl der Präventionsstrategie richtet sich nach dem durch die Bestimmung des Serostatus ermittelten Risiko einer CMV-Erkrankung.

Prophylaxe oder präemptive Therapie? | Bei einer Hochrisikokonstellation (D+/R-) empfiehlt die deutsche Expertengruppe bevorzugt die Prophylaxe. Auch bei anderen Hochrisiko-Patienten kann die Prophylaxe die bevorzugte Strategie sein. Dazu zählen Patienten

- nach kürzlicher Behandlung mit Lymphozytendepletierenden Antikörpern,

- starker Immunsuppression einschließlich Desensibilisierung oder Protokollen bei ABO-inkompatibler Nierentransplantation sowie

- HIV-Patienten [15].

Bei seropositiven Empfängern $(\mathrm{R}+)$ gelten beide Strategien als akzeptabel. Für eine optimale
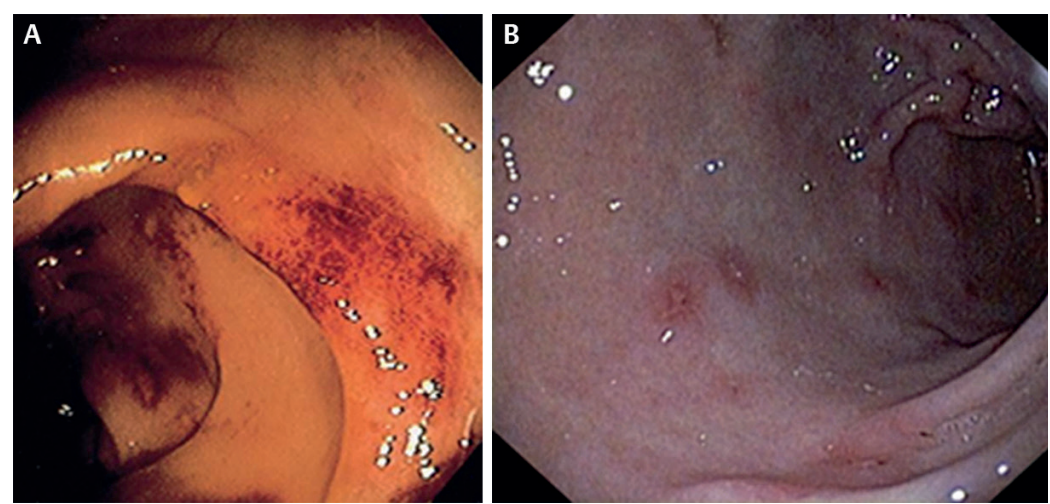

Abb. 2 Gastrointestinale CMV-Erkrankung (A) CMVKolitis und (B) CMV-assoziierte

präemptive Therapie sollte die Viruslast mit einem zentrumspezifischen Protokoll wöchentlich über 3-4 Monate nach der Transplantation gemessen werden. Bei R+-Patienten sollte angesichts der Datenlage [14, 36] die CMV-Prophylaxe bevorzugt werden, wenn ein regelmäßiges Monitoring im Rahmen der präemptiven Therapie nicht gewährleistet werden kann [15] (geringe Übereinstimmung). Das zur Prophylaxe in Deutschland bevorzugte Medikament Valganciclovir $\left(\right.$ Valcyte $^{\circledR}$ ) ist allerdings in Deutschland für seropositive Empfänger bisher nicht zugelassen. Nach Meinung der Expertengruppe ist die Datenlage für den Einsatz aber ausreichend und überzeugend.

Die Expertengruppe weist in diesem Zusammenhang ausdrücklich darauf hin, bei Patienten nach Nierentransplantation neben der CMV-Prophylaxe auch die Empfehlungen zur Prophylaxe von

- Varicella zoster

- Herpes simplex sowie

- Pneumocystis jirovecii zu berücksichtigen.

Nutzen der Präventionsstrategien | Laut einer Cochrane-Analyse reduziert die antivirale CMVProphylaxe die Inzidenz der CMV-Erkrankung und die CMV-assoziierte Mortalität bei Transplantation solider Organe. Die Autoren kommen zu dem Schluss, dass die CMV-Prophylaxe routinemäßig bei CMV-positiven Empfängern $(\mathrm{R}+)$ und bei CMV-negativen Empfängern von CMVpositiven Spendern ( $\mathrm{D}+/ \mathrm{R}-)$ angewendet werden sollte [12]. Einer weiteren Cochrane-Analyse zufolge gilt die präemptive Therapie als effektiv im Vergleich mit Placebo oder einer Standardversorgung. Ihre Wirksamkeit zur Prävention einer CMV-Erkrankung wird aber im Vergleich zur Prophylaxe als weiterhin unklar bezeichnet. Begründet wird dies mit der Heterogenität der ausgewerteten Studien [21].

Dauer der CMV-Prophylaxe I Bei nierentransplantierten Patienten mit der Serokonstellation D+/Rsollte eine 6-monatige CMV-Prophylaxe durchgeführt werden (hohe Übereinstimmung) [13, 15].
Magenulzera nach Nierentransplantation (mit freundlicher Genehmigung von Herrn Professor Kliem, Hann. Münden). 
Da es nach Beendigung der Prophylaxe häufiger zu einer CMV-Primärinfektion kommt, sollte ein besonderes Augenmerk auf die Entwicklung von CMV-Symptomen gelegt werden. Über die Art und Häufigkeit des Monitorings nach Therapieende liegen keine ausreichenden Daten für eine fundierte Empfehlung vor. Die Expertengruppe empfiehlt eine klinische Kontrolle und eine engmaschige Überwachung der Viruslast in den ersten 3-6 Wochen nach Beendigung der Prophylaxe. Bei seropositiven Empfängern $(\mathrm{R}+$ ) sollte die Dauer der Prophylaxe unabhängig vom Donorstatus 3 Monate betragen [15, 14, 36] (hohe Übereinstimmung). Bei starker Immunsuppression (Lymphozyten-depletierende Antikörpertherapie, Desensibilisierungsprotokolle) kann auch bei R+ eine Verlängerung der Prophylaxe auf bis zu 6 Monate erwogen werden.

"Late-onset CMV“ I Das Auftreten einer CMVInfektion bzw. -Erkrankung nach Absetzen der Prophylaxe („late-onset CMV“) ist je nach Risikogruppe unterschiedlich und hängt auch von der Dauer der Prophylaxe ab. Je länger die Prophylaxe durchgeführt wurde, desto geringer ist das Auftreten einer späten CMV-Infektion [13].

Arzneimittel zur CMV-Prophylaxe I Nach Nierentransplantation werden, abhängig von der Möglichkeit einer oralen Medikation,

- Valganciclovir,

- intravenöses Ganciclovir oder

- hochdosiertes Valacyclovir

empfohlen [15]. Die Dosierung der antiviralen Arzneimittel zur CMV-Prophylaxe sollte in Deutschland gemäß der jeweiligen Fachinformation erfolgen und an die Nierenfunktion angepasst werden (Cockcroft-Gault-Formel bzw. Schwartz-Formel bei Kindern und Jugendlichen).

„Mini-Dosing“ I Zum Einsatz der halben Valganciclovir-Dosis (sog. „Mini-Dosing“) mit dem Ziel, Nebenwirkungen und Kosten zu reduzieren, liegen nur limitierte Daten vor. Daher raten die Mitglieder dieser Expertengruppe von einem „Mini-Dosing“ ab. Sie halten prospektive Studien für erforderlich, um die Wirksamkeit zu untersuchen. Alternative Forschungsansätze wie z.B. Maribavir (Proteinkinaseinhibitor) und Letermovir (Inhibitor der viralen Terminase) sind derzeit in der späten klinischen Entwicklung. Es sind jedoch noch weitere Studien erforderlich, um deren die Wirksamkeit und Sicherheit zu bestätigen und zu bewerten.

Toxizitäten | Bevor eine Entscheidung über das Absetzen oder die Modifikation der Immunsuppression (z.B. Mycophenolatmofetil [MMF] oder Mycophenolat-Natrium [MPA]) getroffen wird, sollte zunächst die Komedikation (z.B. Sulfamethoxazol-Trimethoprim) überprüft werden. Diese Entscheidung sollte nur durch das Transplantationszentrum unter Berücksichtigung des individuellen Patientenrisikos erfolgen.
Bei substanzspezifischen Nebenwirkungen sollten Dosisanpassungen der antiviralen CMV-Prophylaxe nur in Einzelfällen und unter individueller Risikoabwägung erfolgen. Valganciclovir sollte bei Unverträglichkeit eher abgesetzt als in reduzierter Dosis gegeben werden, um Ganciclovir-Resistenzen zu vermeiden.

Auswirkungen von Immunsuppressiva auf die CMV-Präventionsstrategie | Bei Transplantatempfängern, die mit mTOR-Inhibitoren wie Sirolimus und Everolimus behandelt werden, wurde in retrospektiven Analysen eine geringere Häufigkeit CMV-assoziierter Komplikationen beschrieben $[3,4,7,9,22,29]$. Es bleibt abzuwarten, ob zukünftige prospektive Studien den zielgerichteten Einsatz dieser Substanzen untermauern und andere CMV-Präventionsstrategien ermöglichen.

\section{Therapie}

Arzneimittel I Bei nicht-lebensbedrohlichen CMV-Erkrankung von Erwachsenen werden

- Valganciclovir und

- intravenöses Ganciclovir

als Therapie der Wahl empfohlen $[1,15]$ (hohe Übereinstimmung). Valganciclovir soll bevorzugt eingesetzt werden. Ausnahmen:

- lebensbedrohliche CMV-Erkrankung und

- Situationen, bei denen eine schlechte Bioverfügbarkeit und Non-Compliance mit der Medikation wahrscheinlich sind [15] (hohe Übereinstimmung).

Ein Wechsel zwischen beiden Substanzen (z. B. von Ganciclovir i.v. auf Valganciclovir) kann ohne Unterbrechung der Dosierung durchgeführt werden [15] (hohe Übereinstimmung). In Deutschland ist Valganciclovir bei Patienten nach Nierentransplantation nicht zur Therapie einer CMV-Infektion zugelassen. Nach Meinung der Expertengruppe sprechen aber die Datenlage und insbesondere die Praktikabilität für den oralen Einsatz. Die Anwendung von oralem Ganciclovir, Aciclovir oder Valaciclovir wird nicht empfohlen [15] (hohe Übereinstimmung).

Dosis und Behandlungsdauer I Die Behandlung erfolgt mit folgender Dosierung:

- Ganciclovir i.v.: $5 \mathrm{mg} / \mathrm{kg}, 2 \times$ tgl.

- Valganciclovir: $900 \mathrm{mg}, 2 \times$ tgl.

Sie sollte so lange dauern, bis in Wochenabstand mindestens zweimal eine Viruseradikation mittels PCR-basierten Testverfahren im peripheren Blut nachgewiesen werden kann [15] (hohe Übereinstimmung). Die Grenzwerte der Viruslast zum Absetzen der Therapie sind derzeit nicht standardisiert. Die Beendigung der Therapie sollte sich nach der Klinik und der Erfahrung mit den verwendeten Testverfahren richten. Die Expertengruppe ist der Meinung, dass bei fehlender adäquater Absenkung der Viruslast eher die emp- 
fohlene Dosierung von Ganciclovir i.v. überschritten werden sollte, zumal dies im Falle einer möglichen Ganciclovir-Resistenz auch zunächst die Therapie der Wahl wäre [10]. Die Nierenfunktion sollte während der CMV-Therapie häufig überwacht und die antivirale Dosierung auf Basis der Fachinformation anhand der CockcroftGault-Formel angepasst werden (entsprechende Dosisempfehlungen für Erwachsene mit eingeschränkter Nierenfunktion siehe Fachinformation Valcyte ${ }^{\circledR}$ und Cymeven ${ }^{\circledR}$ ).

Toxizitäten während der CMV-Therapie I Treten Nebenwirkungen auf (z. B. Leukozytopenie), sollte eine Dosisreduktion von Valganciclovir oder Ganciclovir in der Regel vermieden werden, um die Entstehung von CMV-Resistenzen nicht zu begünstigen [15] (hohe Übereinstimmung). Neben substanzspezifischen Ursachen kommen auch andere andere Gründe für die Leukozytopenie in Betracht, die möglichst durch Dosisreduktionen bzw. -modifikationen anderer myelosuppressiver Therapien (z.B. Mycophenolsäure, Allopurinol) oder der antibiotischen Prophylaxe (z.B. Sulfamethoxazol-Trimethoprim) adressiert werden sollten. Eine Leukozytopenie ist gelegentlich - auch nach Therapiebeginn - durch die aktive CMVInfektion selbst bedingt und erfordert möglicherweise eine noch konsequentere antivirale Therapie. In Ausnahmefällen ist vor dem Absetzen oder der Dosisreduktion der antiviralen Medikation die Gabe von Granulozyten-Kolonie-stimulierendem Faktor (G-CSF) in Betracht zu ziehen [15].

Vorgehensweise bei einem CMV-Rezidiv | Bei einem CMV-Rezidiv gelten die gleichen Behandlungsoptionen wie bei einer CMV-Ersterkrankung [15] (hohe Übereinstimmung).

Ganciclovir-Resistenz I Eine Ganciclovir-Resistenz kann asymptomatisch, schwer oder tödlich verlaufen $[2,18]$. Der Verdacht auf eine Ganciclovir-Resistenz liegt vor, wenn bei einer prolongierten antiviralen Therapie die Viruslast oder die klinischen Symptome nicht zurückgehen (hohe Übereinstimmung). Eine prolongierte Therapie bedeutet eine kumulative antivirale Arzneimittelexposition über mindestens 6 Wochen, einschließlich einer seit mehr als 2 Wochen bestehenden Therapie unter voller Dosis (i.v. Ganciclovir $5 \mathrm{mg} / \mathrm{kg}, 2 \times$ täglich) zum Zeitpunkt der Bewertung. Die Inzidenz von GanciclovirResistenzen bei D+/R--Empfängern nach Nierentransplantation liegt nach einer CMV-Prophylaxe über 100-200 Tage bei 0-3\% [2]. Besteht der Verdacht einer Ganciclovir-Resistenz sollte in Referenzlaboren eine Genotypisierung der viralen Gene UL97 und UL54 durchgeführt werden. Als Probenmaterial dienen meist Blut (Vollblut, Plasma, Leukozyten) oder Gewebeproben.

Behandlung bei Ganciclovir-Resistenz | Zunächst sollte abgeklärt werden, ob eine Dosisreduktion der Immunsuppressiva möglich ist. Bei einer CMV UL97-Mutation kann Cidofovir zur Behandlung eingesetzt werden [26]. Außerdem bestehen folgende Behandlungsoptionen:

- bei schwerer Erkrankung: Foscavir

- bei nicht-schwerer Erkrankung: Ganciclovir i.v. als Hochdosistherapie (bis zu 10 mg / kg 2 × täglich, entspricht der doppelten Standarddosis)

Wenn Foscavir vermieden werden soll, können alternative Therapieoptionen bei nicht-schwerer Erkrankung sein [10]:

- nierenfunktionsadaptierte Hochdosistherapie mit Ganciclovir i.v. (7,5-10 mg/ kg alle $12 \mathrm{~h})$

- Valganciclovir oral (1350-1800 mg 2× tgl)

\section{Konsequenz für Klinik und Praxis}

- Vor der Transplantation sollte die CMV-Serologie des Spenders (D) und des Empfängers (R) bestimmt werden. Bei einer Hochrisikokonstellation ( $D+/ R$-) wird eine CMV-Prophylaxe empfohlen.

- Beim Einsatz von Valganciclovir zur CMV-Prophylaxe sollte die Dosierung regelmäßig überprüft und an die Nierenfunktion angepasst werden.

- In den ersten 3-6 Wochen nach Beendigung der CMV-Prophylaxe wird eine klinische Kontrolle und eine engmaschige Überwachung der Viruslast empfohlen.

- Bei einer Gewebs-invasiven CMV-Erkrankung kann die CMV-PCR im Blut negativ ausfallen.

\section{Online-Zusatzmaterial}

Informationen zum CMV-Management bei Kindern finden Sie im Online-Zusatzmaterial unter

http://dx.doi.org/10.1055/s-0041-100777

Vollständiges Literaturverzeichnis unter http://dx.doi.org/10.1055/s-0041-100777

\section{Expertentreffen}

„State of the Art: CMV-Management bei Nierentransplantation“ am 8. Juli 2013, Frankfurt/Main, unterstützt von der Roche Pharma AG, Grenzach-Wyhlen.

\section{Danksagung}

Wir danken Frau Dr. Schäfer (MEDICAL COMMUNICATION CONSULTING) für die editorielle Unterstützung bei der

Erstellung des Manuskriptes.

\section{Interessenkonflikte}

VK hat Studienunterstützungen, Honorare für Vorträge, Reisekostenübernahmen und Unterstützungen für die Anfertigung und Präsentation medizinisch / wissenschaftlicher Vorträge von den Firmen Astellas, Bristol-Myers Squibb, Genzyme, Novartis, Fresenius, Roche, Raptor und Pfizer erhalten.

MS hat Forschungsunterstützungen, Honorare für Vorträge, Reisekostenübernahmen von den Firmen Astellas, Genzyme, Novartis, Roche, Qiagen und Pfizer erhalten. MS ist Mitgründerin der RedFlag Diagnostics $\mathrm{GmbH}$.

MN hat Studienunterstützungen, Honorare für Vorträge, Reisekostenübernahmen von den Firmen Astellas, Genzyme, Novartis, Roche und Bristol-Myers Squibb und Forschungsunterstützungen von Qiagen erhalten.

BT hat Studienunterstützungen, Honorare für Vorträge, Reisekostenübernahmen und Unterstützungen für die Anfertigung und Präsentation medizinisch/ wissenschaftlicher Vorträge von den Firmen Astellas, Bristol-Myers Squibb, Genzyme, Novartis, Roche und Pfizer erhalten. KB übt Beratungstätigkeiten für die Firmen Bristol-Myers Squibb, Veloxis-Pharma, Novartis Pharma, Effimune Pharma und Pfizer aus; KB hat Studienunterstützungen, Honorare für Vorträge, Reisekostenübernahmen und Unterstützungen für die Anfertigung und Präsentation medizinisch / wissenschaftlicher Vorträge von den Firmen AiCuris, Astellas, Bristol-Myers Squibb, Hexal, Veloxis Pharma, Chiesi, Novartis Pharma; Siemens, Fresenius, Gambro, Roche AG und Pfizer erhalten

IAH, MSch und BH haben von der Firma Roche Reisekostenunterstützung und Honorar für die aktive Teilnahme an einem Advisory Board erhalten

OW hat Studienunterstützungen, Honorare für Vorträge, Reisekostenübernahmen und Unterstützungen für die Anfertigung und Präsentation medizinisch / wissenschaftlicher Vorträge von den Firmen Amgen, Astellas, Bristol-Myers Squibb, Chiesi, Novartis, Roche, Pfizer und Sanofi erhalten. 
Literatur

1 Asberg A, Humar A, Jardine AG et al. Long-term outcomes of CMV disease treatment with valganciclovir versus IV ganciclovir in solid organ transplant recipients. Am J Transplant 2009; 9: 1205-1213

2 Boivin G, Goyette N, Farhan M et al. Incidence of cytomegalovirus UL97 and UL54 amino acid substitutions detected after 100 or 200 days of valganciclovir prophylaxis. J Clin Virol 2012; 53: 208-213

3 Brennan DC, Legendre C, Patel D et al. Cytomegalovirus incidence between everolimus versus mycophenolate in de novo renal transplants: pooled analysis of three clinical trials. Am J Transplant 2011; 11: 2453-2462

4 Cervera C, Fernandez-Ruiz M, Valledor A et al. Epidemiology and risk factors for late infection in solid organ transplant recipients. Transpl Infect Dis 2011; 13: 598-607

5 Dickenmann MJ, Cathomas G, Steiger J et al. Cytomegalovirus infection and graft rejection in renal transplantation. Transplantation 2001; 71: 764-767

6 Eid AJ, Arthurs SK, Deziel PJ, Wilhelm MP, Razonable RR. Clinical predictors of relapse after treatment of primary gastrointestinal cytomegalovirus disease in solid organ transplant recipients. Am J Transplant 2010; 10: 157-161

7 Ekberg H, Bernasconi C, Noldeke J et al. Cyclosporine, tacrolimus and sirolimus retain their distinct toxicity profiles despite low doses in the Symphony study. Nephrol Dial Transplant 2010; 25: 2004-2010

8 Fachinformation. Valcyte ${ }^{\circledR} 50 \mathrm{mg} / \mathrm{ml}$ Pulver zur Herstellung einer Lösung zum Einnehmen (Mono), Stand: April 2013. FachInfo-Service; 2013

9 Fortun J, Martin-Davila P, Pascual J et al. Immunosuppressive therapy and infection after kidney transplantation. Transpl Infect Dis 2010; 12: 397-405

10 Gracia-Ahufinger I, Gutierrez-Aroca J, Cordero E et al. Use of high-dose ganciclovir for the treatment of cytomegalovirus replication in solid organ transplant patients with ganciclovir resistance-inducing mutations. Transplantation 2013; 95: 1015-1020

11 Halme L, Lempinen M, Arola J et al. High frequency of gastroduodenal cytomegalovirus infection in liver transplant patients. APMIS 2008; 116: 99-106

12 Hodson EM, Ladhani M, Webster AC et al. Antiviral medications for preventing cytomegalovirus disease in solid organ transplant recipients. Cochrane Database Syst Rev 2013; 2: CD003774

13 Humar A, Limaye AP, Blumberg EA et al. Extended valganciclovir prophylaxis in $\mathrm{D}+/ \mathrm{R}$ - kidney transplant recipients is associated with long-term reduction in cytomegalovirus disease: two-year results of the IMPACT study. Transplantation 2010; 90: 1427-1431

14 Kliem V, Fricke L, Wollbrink T et al. Improvement in long-term renal graft survival due to CMV prophylaxis with oral ganciclovir: results of a randomized clinical trial. Am J Transplant 2008; 8: 975-983

15 Kotton CN, Kumar D, Caliendo AM et al. Updated international consensus guidelines on the management of cytomegalovirus in solid-organ transplantation. Transplantation 2013; 96: 333-360

16 Kranz B, Vester U, Wingen AM et al. Acute rejection episodes in pediatric renal transplant recipients with cytomegalovirus infection. Pediatr Transplant 2008; 12: 474-478

17 Lautenschlager I, Halme L, Hockerstedt K et al. Cytomegalovirus infection of the liver transplant: virological, histological, immunological, and clinical observations. Transpl Infect Dis 2006; 8: 21-30

18 Le Page AK, Jager MM, Iwasenko JM et al. Clinical aspects of cytomegalovirus antiviral resistance in solid organ transplant recipients. Clin Infect Dis 2013; 56 : 1018-1029
19 Ljungman P, Griffiths P, Paya C. Definitions of cytomegalovirus infection and disease in transplant recipients. Clin Infect Dis 2002; 34: 1094-1097

20 McLaughlin K, Wu C, Fick G et al? Cytomegalovirus seromismatching increases the risk of acute renal allograft rejection. Transplantation 2002; 74: 813-816

21 Owers DS, Webster AC, Strippoli GF et al. Pre-emptive treatment for cytomegalovirus viraemia to prevent cytomegalovirus disease in solid organ transplant recipients. Cochrane Database Syst Rev 2013; 2: CD005133

22 Ozaki KS, Camara NO, Nogueira E et al. The use of sirolimus in ganciclovir-resistant cytomegalovirus infections in renal transplant recipients. Clin Transplant 2007; 21: 675-680

23 Pescovitz MD. Prevention and treatment of cytomegalovirus disease in solid organ transplant recipients: the clinical and economic impact of evolving strategies. Introduction. Am J Health Syst Pharm 2003; 60(Suppl 8): S3-S4

24 Pilmore H, Pussell B, Goodman D. KHA-CARI guideline: cytomegalovirus disease and kidney transplantation. Nephrology 2011; 16: 683-687

25 Ritter M, Schmidt T, Dirks J et al. Cytomegalovirusspecific T cells are detectable in early childhood and allow assignment of the infection status in children with passive maternal antibodies. Eur ] Immunol 2013; 43: 1099-1108

26 Robert Koch-Institut. Epidemiologisches Bulletin Nr. 3, Januar 2014

27 Sagedal S, Hartmann A, Nordal KP et al. Impact of early cytomegalovirus infection and disease on long-term recipient and kidney graft survival. Kidney Intern 2004; 66: 329-337

28 Sagedal S, Nordal KP, Hartmann A et al. A prospective study of the natural course of cytomegalovirus infection and disease in renal allograft recipients. Transplantation 2000; 70: 1166-1174

29 San Juan R, Aguado JM, Lumbreras C et al. Impact of current transplantation management on the development of cytomegalovirus disease after renal transplantation. Clin Infect Dis 2008; 47: 875-882

30 Sester M, Gärtner BC, Sester U. Monitoring of CMV-specific T-cell levels after organ transplantation. Lab Med 2008; 32: 121-130

31 Smith JM, Corey L, Bittner R et al Subclinical viremia increases risk for chronic allograft injury in pediatric renal transplantation. J Am Soc Nephrol 2010; 21: 1579-1586

32 Stitt N. Infection in the Transplant Recipient. . Medscape Online 2003; http://www.medscape.com/ viewarticle/451788. Letzter Zugriff: 18. 02. 2015

33 Tong CY, Bakran A, Peiris JS et al. The association of viral infection and chronic allograft nephropathy with graft dysfunction after renal transplantation. Transplantation 2002; 74: 576-578

34 van Son WJ, de Maar EF, van Der Bij W et al. Overcoming the problem of cytomegalovirus infection after organ transplantation: calling for Heracles? Intervirology $1999 ; 42: 285-290$

35 Westall GP, Michaelides A, Williams T] et al. Human cytomegalovirus load in plasma and bronchoalveolar lavage fluid: a longitudinal study of lung transplant recipients. J Infect Dis 2004; 190: 1076-1083

36 Witzke O, Hauser IA, Bartels M et al. Valganciclovir prophylaxis versus preemptive therapy in cytomegalovirus-positive renal allograft recipients: 1 -year results of a randomized clinical trial. Transplantation 2012; 93 : 61-68 